\title{
CX3CR1-dependent subretinal microglia cell accumulation is associated with cardinal features of age-related macular degeneration
}

\author{
Christophe Combadière,1,2,3 Charles Feumi,4,5,6 William Raoul,4,5,6 Nicole Keller,4,5,6 \\ Mathieu Rodéro, ${ }^{1,2}$ Adeline Pézard, ${ }^{1}$ Sophie Lavalette, ,4,5,6 Marianne Houssier, 4,5,6 Laurent Jonet, ${ }^{4,5,6}$ \\ Emilie Picard,4,5,6 Patrice Debré,1,2,3 Mirna Sirinyan,7 Philippe Deterre,,1,2 Tania Ferroukhi,1 \\ Salomon-Yves Cohen, ${ }^{8}$ Dominique Chauvaud, 9 Jean-Claude Jeanny, ${ }^{4,5,6}$ Sylvain Chemtob, 7 \\ Francine Behar-Cohen, $4,5,6,9$ and Florian Sennlaub4,5,6
}

\begin{abstract}
${ }^{1}$ INSERM U543, Laboratoire d'Immunologie Cellulaire, Paris, France. 'Université Pierre et Marie Curie, Laboratoire d'informatique de Paris 6, Paris, France. ${ }^{3}$ AP-HP, Groupe Hospitalier Pitié-Salpétrière, Service d'Immunologie, Paris, France. ${ }^{4}$ INSERM U872, Paris, France. ${ }^{5}$ Centre de Recherche des Cordeliers, Université Pierre et Marie Curie, Laboratoire d'informatique de Paris 6, Paris, France. 6Université Paris Descartes, UMR S 872, Paris, France. 7Department of Pediatrics, Ophthalmology and Pharmacology, Research Center, Hôpital Ste Justine, Montréal, Québec, Canada. ${ }^{8}$ Centre d'Angiographie et de Laser, Paris, France. ${ }^{9}$ AP-HP, Hôtel Dieu, Service d'Ophtalmologie, Centre de Recherche Ophtalmologique, Paris, France.
\end{abstract}

\begin{abstract}
The role of retinal microglial cells (MCs) in age-related macular degeneration (AMD) is unclear. Here we demonstrated that all retinal MCs express CX3C chemokine receptor 1 (CX3CR1) and that homozygosity for the CX3CR1 M280 allele, which is associated with impaired cell migration, increases the risk of AMD. In humans with AMD, MCs accumulated in the subretinal space at sites of retinal degeneration and choroidal neovascularization (CNV). In CX3CR1-deficient mice, MCs accumulated subretinally with age and albino background and after laser impact preceding retinal degeneration. Raising the albino mice in the dark prevented both events. The appearance of lipid-bloated subretinal MCs was drusen-like on funduscopy of senescent mice, and CX3CR1-dependent MC accumulation was associated with an exacerbation of experimental CNV. These results show that CX3CR1-dependent accumulation of subretinal MCs evokes cardinal features of AMD. These findings reveal what we believe to be a novel pathogenic process with important implications for the development of new therapies for AMD.
\end{abstract}

\section{Introduction}

Age-related macular degeneration (AMD) is the leading cause of vision loss in elderly people in the industrialized countries (1). Its most prominent pathologic features are lesions involving the retinal pigment epithelium (RPE) and Bruch's membrane (BM), photoreceptor degeneration (2), and, in the most aggressive cases, choroidal neovascularization (CNV). Early AMD is characterized by yellowish-white dots called drusen, located on BM, partially covered by the RPE and clinically visible with funduscopy (3). Composed of lipids and glycoproteins (4), drusen are believed to be formed by extracellular deposit of materials or by transformed degenerating RPE cells (5). Controversy continues to surround their pathogenesis, as well as the causes of AMD.

Epidemiological studies have helped to identify key factors in the pathogenesis of AMD. Age (1) and family history (3) are the principal predisposing factors. Genes with polymorphisms associated with AMD include apolipoprotein E (6), superoxide dismutase (7), complement factor $\mathrm{H}(8,9)$, and CX3C cheomkine receptor 1 (CX3CR1) (10).

Some of the features of human AMD have been observed in transgenic mouse models. Notably, a deficiency in macrophage

Nonstandard abbreviations used: AMD, age-related macular degeneration; BM, Bruch's membrane; CCL2, CC chemokine ligand 2; CCR2, CC chemokine receptor 2; $\mathrm{CNV}$, choroidal neovascularization; CX3CL1, CX3C chemokine ligand 1; CX3CR1, CX3C chemokine receptor 1; MC, microglial cell; OS, outer segment; RPE, retinal pigment epithelium; SrMC, subretinal MC.

Conflict of interest: The authors have declared that no conflict of interest exists. Citation for this article: J. Clin. Invest. 117:2920-2928 (2007). doi:10.1172/JCI31692. recruitment through a CC chemokine ligand 2- (CCL2-) and CC chemokine receptor 2-dependent (CCR2-dependent) pathway from the choroidal circulation may prevent the clearance of accumulating debris in $\mathrm{BM}$; this has been proposed as a mechanism for drusen genesis (11). Moreover, CCL2- and CCR2-dependent macrophage recruitment has previously been shown to participate in CNV development (12). Studying the expression of the chemokine receptors CCR2 and CX3CR1 (receptor for CX3C chemokine ligand 1; CX3CL1) identifies 2 functional subsets of murine blood monocytes: inflammatory monocytes, which express both receptors, and noninflammatory monocytes, which express only CX3CR1 (13). Microglial cells (MCs) express CX3CR1 and play a role in controlling microglial neurotoxicity in the brain (14). We postulated that CX3CR1 is expressed on MCs in the photoreceptor layer in AMD (15) and participates in AMD development and progression by controlling retinal MC redistribution. Accordingly, we analyzed CX3CR1 polymorphisms and expression in human AMD and studied the eyes of 2 independently generated CX3CR1 knockout mouse strains in aging- and laser-induced CNV. We found that CX3CR1 invalidation led to subretinal MC (SrMC) accumulation, which was associated with drusen-like lesions, retinal degeneration, and exacerbated neovascularization, cardinal features of AMD.

\section{Results}

CX3CR1 polymorphism and AMD. To evaluate the role of the chemokine receptor CX3CR1 in AMD, we studied the distribution of 2 previously reported variants of CX3CR1 (T280M and V249I) in 
Table 1

Distribution of CX3CR1 T280M in GAMD and reference studies

\begin{tabular}{lccccc}
\hline \multicolumn{5}{c}{ Study (N) } \\
Distribution & GAMD (284) & DETER (520) & ECTIM (2,212) & FHS (1,859) & GENIC (929) \\
TT (no.) & $64.1 \%(182)$ & $76.0 \%(395)$ & $68.9 \%(1,523)$ & $67.5 \%(1,254)$ & $70.2 \%(652)$ \\
TM (no.) & $29.9 \%(85)$ & $21.7 \%(113)$ & $28.5 \%(631)$ & $29.6 \%(550)$ & $26.7 \%(248)$ \\
MM (no.) & $6.0 \%(17)$ & $2.3 \%(12)$ & $2.6 \%(58)$ & $3.0 \%(55)$ & $3.1 \%(29)$ \\
Frequency & 0.21 & 0.13 & 0.17 & 0.18 & 0.16 \\
$P$ & - & 0.0098 & 0.0033 & 0.0139 & 0.033 \\
Odds ratio (95\% & - & 2.695 & 2.365 & 2.088 & 1.976 \\
confidence interval) & & $(1.268$ to 5.728$)$ & $(1.357$ to 4.121$)$ & $(1.194$ to 3.652) (1.069 to 3.652) \\
\end{tabular}

Distribution of patients homozygous for threonine (TT) and methionine (MM), common and rare alleles, respectively, at position 280 , as well as patients heterozygous for threonine/methionine (TM) at position 280 . N, total number of participants. AAllelic frequency of allele M. $P$ values and odds ratios were determined by comparing the frequency of the MM genotype with the frequency of T carriers. The DETER (P. Deterre, unpublished observations), ECTIM (16), FHS (17), and GENIC (18) studies were used as reference studies.

a sample of elderly individuals with AMD (Genetics of Age-related Macular Degeneration study; GAMD; Table 1 and Supplemental Table 1; supplemental material available online with this article; doi:10.1172/JCI31692DS1). The frequencies of CX3CR1 polymorphisms did not deviate from Hardy-Weinberg equilibrium among AMD cases or subjects from reference studies (data not shown). Table 1 shows the distribution of the CX3CR1 T280M genotypes among the subjects from the DETER study (P. Deterre, unpublished observations) and the previously published Etude Cas-Témoin de l'Infarctus du Myocarde (ECTIM; ref. 16), Offspring Cohort of the Framingham Heart Study (FHS; ref. 17), and Étude du Profil Génétique de l'Infarctus Cérébral (GENIC; ref. 18) studies used as references. Homozygosity for the M280 allele was consistently more frequent in studies of AMD than of other diseases. Odds ratios associated with homozygosity for the rare allele were therefore computed with genotypes carrying the more frequent allele as the reference groups. M280 homozygosity was associated with an increased risk of AMD, with odds ratios ranging from 1.97 to 2.695 (95\% confidence intervals, $1.069-3.652$ and $1.268-5.728$, respectively). This association was independent of age, and disease severity was not associated with the polymorphism. The similarity of the genotype distribution of the CX3CR1 V249I alleles among AMD patients and subjects from reference studies (Supplemental Table 1) indicated that this polymorphism had no significant effect on AMD risk.

CX3CR1 expression in AMD. To understand the mechanism by which CX3CR1 alterations may contribute to AMD development, we analyzed CX3CR1 expression in eyes of healthy donors and subjects with AMD (Figure 1). CX3CR1 positivity (red staining) was found in cells of the inner retina. Double labeling for CX3CR1 (green fluorescence) and the vascular endothelial cell marker ulex (red fluorescence) showed the ramified morphology of cells expressing CX3CR1 and their closeness to the retinal vasculature in the healthy eye (Figure 1A, inset). No CX3CR1 positivity was detected in photoreceptors or RPE cells in healthy donor eyes. In the macula of age-matched patients with AMD, we observed localized RPE disruption and clumping as well as photoreceptor degeneration (Figure 1B). In the affected zones, CX3CR1-positive cells were found ectopically in the outer retina and in the subretinal space, as well as in the perivascular retinal MCs in the inner retina (Figure 1B). Some of these cells in the outer retina were very large and bloated (Figure 1B, inset).
All CX3CR1-positive cells (Figure 1C) overlapped precisely with cells expressing the microglial marker CD18 (Figure $1 \mathrm{D})$, and vice versa (Figure 1E). Furthermore, CX3CR1 deposits were found in drusen, specifically in the CD18-positive dendritic cells interposed between the choroidal vessels (Figure 1F). CX3CR1positive cells were also found in close contact with CNV (Figure 1G).

The M280 polymorphism leads to migrational defect. We previously showed that only the membrane-anchored form of CX3CL1 functionally discriminates between the 2 allelic isoforms of CX3CR1 (19): monocytes bearing the CX3CR1-IM allele adhere more strongly than cells expressing only the CX3CR1-VT variant. In contrast, their chemotactic response to CX3CL1 does not distinguish between the 2 monocyte populations. To gain insight into the ability of cells expressing different variants of CX3CR1 to migrate in response to chemoattractants present in the retina, we studied the in vitro chemotaxis of monocytes in response to CCL2 through porous membranes with and without a CX3CL1 coating. Monocytes from individuals homozygous for the CX3CR1-VT or CX3CR1-IM isoforms migrated equally well to CCL2 (Figure 2A). CCL2-specific chemotactic responses of monocytes from individuals homozygous for the VT variant were similar whether or not the filter-bound CX3CR1 was present (Figure 2A), with chemotactic index ratios (index without CX3CL1/ index with CX3CL1) of $1.1 \pm 0.1$. In contrast, monocytes from individuals homozygous for CX3CR1-IM variants migrated in smaller numbers to the lower chamber in response to CCL2 when anchored CX3CL1 was present than when it was not (Figure 2A). This inhibition reached $48 \% \pm 11 \%$ (Figure $2 \mathrm{~B}$ ). These data are consistent with our previous work (19) and indicate that chemotaxis of monocytes from individuals with IM genotypes is impaired in the presence of bound CX3CL1.

SrMCs accumulate in $\mathrm{CX} 3 \mathrm{CR}^{-/}$mice with age and develop retinal degeneration. Because CX3CR1 was expressed on human MC (Figure 1) and because a CX3CR1 polymorphism that impairs cell migration was associated with risk of AMD (Table 1 and ref. 10), we explored the role of CX3CR1 in retinal homeostasis using mice deficient in CX3CR1 gene (20). We first identified the site of CX3CR1 expression by using CX3CR1GFP/GFP (CX3CR1-deficient with GFP driven by CX3CR 1 promoter) and CX3CR1 $1^{+/ G F P}$ mice of different ages. In 18-month-old CX3CR1 $1^{+/ G F P}$ and CX3CR $1^{\text {GFP/GFP }}$ mice, GFP-positive cells abutted retinal endothelial cells (red fluorescence, BSA-1; Figure 3, A and B). Confocal images of the outer plexiform layer of retinal flatmounts displayed dense networks of ramified MCs of similar density in CX3CR $1^{+/ G F P}$ and CX3CR1 $1^{\mathrm{GFP} / \mathrm{GFP}}$ animals $\left(266 \pm 19\right.$ and $305 \pm 27 \mathrm{MCs} / \mathrm{mm}^{2} ; n=4$ per group; Figure 3, C and D), indicating that all MCs expressed CX3CR1 and that homing of retinal MCs was CX3CR1 independent. The expression of the reporter gene in individual MCs was similar in CX3CR1 $1^{+/ G F P}$ and CX3CR $1^{\text {GFP/GFP }}$ mice. The absence of CX3CR1 expression in neurons, astrocytes, Müller cells, and RPE demonstrated that MCs were the only CX3CR1-posititive cells in the retina. 

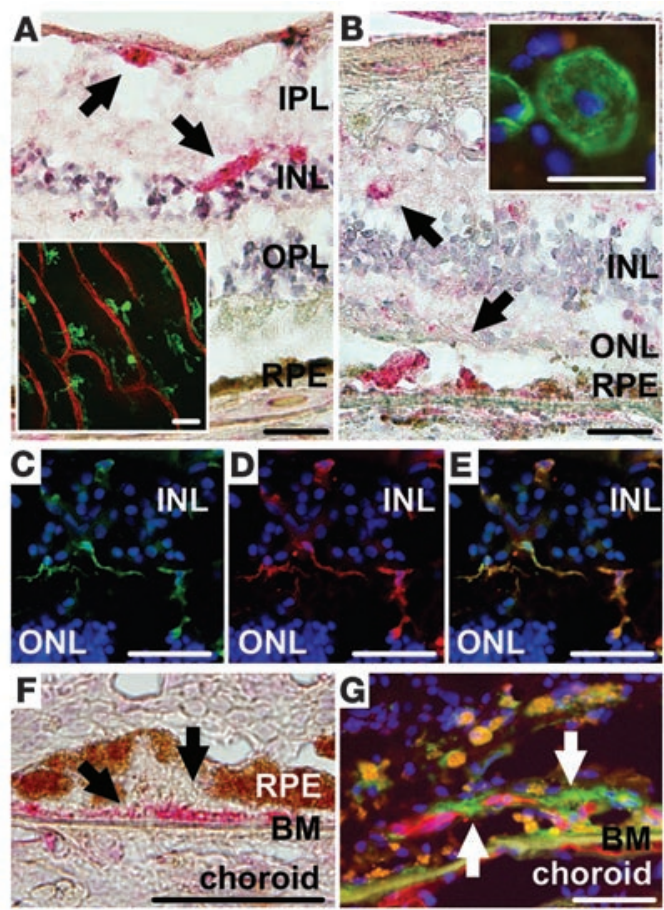

On RPE flatmounts of 18-month-old CX3CR1 $1^{+/ G F P}$ mice (Figure $3 \mathrm{E}$ ), GFP-positive SrMCs were seen occasionally, as previously reported (21), whereas in CX3CR1 GFP/GFP mice, SrMCs juxtaposed to RPE (Figure 3B, arrows) accumulated markedly (Figure 3F) as a function of age (Figure $3 \mathrm{G}$ ). In $\mathrm{CX} 3 \mathrm{CR} 1^{-/}$mice without the GFP transgene, CD11b-positive SrMCs accumulated in a manner similar to that of the analogous CX3CR $1^{\mathrm{GFP} / \mathrm{GFP}}$ animals (data not shown). Histological sections of 18 -month-old CX3CR1 $1^{+/+}$(Figure $3 \mathrm{H}$ ) and $\mathrm{CX} 3 \mathrm{CR} 1^{-/}$(Figure 3I) mice revealed substantial degeneration of the outer retina in the knockout mice. Measurements of the photoreceptor cell layer showed significant thinning (40\%) in CX3CR1 1 - mice (Figure 3J).

SrMC accumulation is associated with retinal degeneration in albino $\mathrm{CX} 3 \mathrm{CR} 1^{-/-}$mice. Light has been suggested to be a contributing factor in the genesis of AMD (22). In conventional light conditions in wild-type albino animals, SrMCs accumulate as a function of ambient light intensity; this accretion is inhibited by light restriction

\section{Figure 2}

The M280 polymorphism leads to CX3CR1 dysfunction and impaired migration. (A) Representative CCL2-dependent migration through CX3CL1-coated filters of monocytes from individuals homozygous for the 2 extreme haplotypes of CX3CR1 (VT and IM). Flow cytometry was used to count the number of CD14-positive cells that migrated into the lower chamber. Each data point is the mean \pm SEM of 3 different determinations. (B) Genetic variations of CX3CR1-impaired CCL2-dependent migration through filters coated with CX3CL1. Monocytes from individuals homozygous for CX3CR1-IM variants $(n=5)$ have less CCL2-dependent chemotactic ability in the presence of a CX3CL1-coated filter than do monocytes from individuals homozygous for CX3CR1-VT variants $(n=8)$. Migration ability is expressed as the ratio of the chemotactic index $(\mathrm{Cl})$ in response to CCL2 of monocytes migrating through a filter with or without a CX3CL1 coating (chemotactic index without CX3CL1 relative to chemotactic index with CX3CL1). ${ }^{\star \star \star} P=0.0006$.

\section{Figure 1}

CX3CR1 expression in AMD. (A) CX3CR1 (fast red labeling) was expressed in the inner retina (arrows); no CX3CR1-positive cells were found in the outer nuclear layer (ONL), photoreceptor OSs, or RPE. Inset: Confocal images of the nerve fiber layer of double-labeled retinal flatmounts showed the ramified morphology of cells expressing CX3CR1 (green) and their close proximity to the retinal vasculature (Ulex red) in a healthy eye. (B) In affected zones of the macula of age-matched subjects with AMD, additional CX3CR1-positive cells (arrows) were found in and below the outer nuclear layer that contains the photoreceptors. Inset: These cells were large and bloated (green, CX3CR1; blue, DAPI). (C-E) CX3CR1-expressing cells (C) were ramified cells that also labeled positive for the MC marker CD18 (red, D); merged image is shown in E. (F) Drusen contained acellular CX3CR1 deposits (arrows). (G) CX3CR1-positive cells were in close physical contact with CNV (arrows). Results are representative for immunohistochemistry from 4 eyes with AMD and 3 age-matched control eyes. INL, inner nuclear layer; IPL, inner plexiform layer. Scale bar: $50 \mu \mathrm{m}$ (A-G and $\mathbf{A}$, inset); $20 \mu \mathrm{m}$ (B, inset).

(21). Accordingly, we found that 1 -month-old CX3CR1 $1^{+/+}$albino mice raised in normal light conditions (12-h light/12-h dark cycle) exhibited some CD11b-positive SrMCs (green fluorescence) adjacent to the RPE (phalloidin red fluorescence; Figure 4A), but these were significantly more abundant in age-matched CX3CR1-/- mice (Figure 4B). The number of SrMC increased as a function of age in wild-type and knockout mice, but the accumulation was accentuated in the latter (Figure 4C). Moreover, the CX3CR1 $1^{-/}$BALB/c mice housed in complete darkness throughout the experiment accumulated significantly fewer SrMCs at 2 months than did the CX3CR1 $1^{-/}$mice raised in ambient light (Figure 4C).

Histological sections of 4-month-old CX3CR1 ${ }^{+/+}$(Figure 4D) and CX3CR1 $1^{-/}$(Figure 4E) mice showed marked degeneration of the outer retina in the latter; this degeneration was not observed in the dark-raised knockout mice (Figure 4F). Quantification of photoreceptor cell layer thickness showed a slight (20\%) but significant thinning at 2 months and complete degeneration at 4 months in the knockout mice, both of which were prevented by light restriction (Figure 4G).

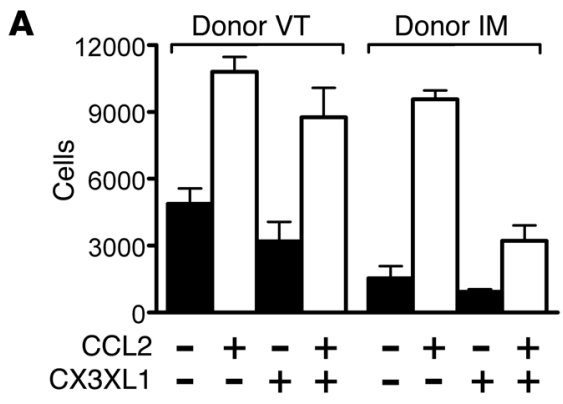

B

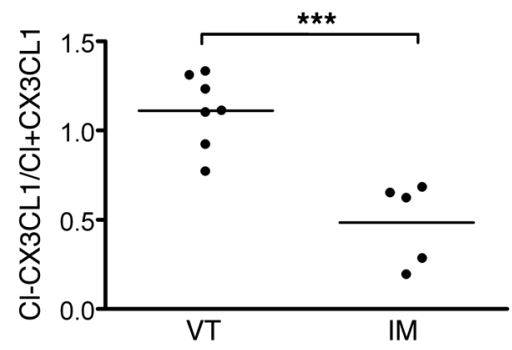



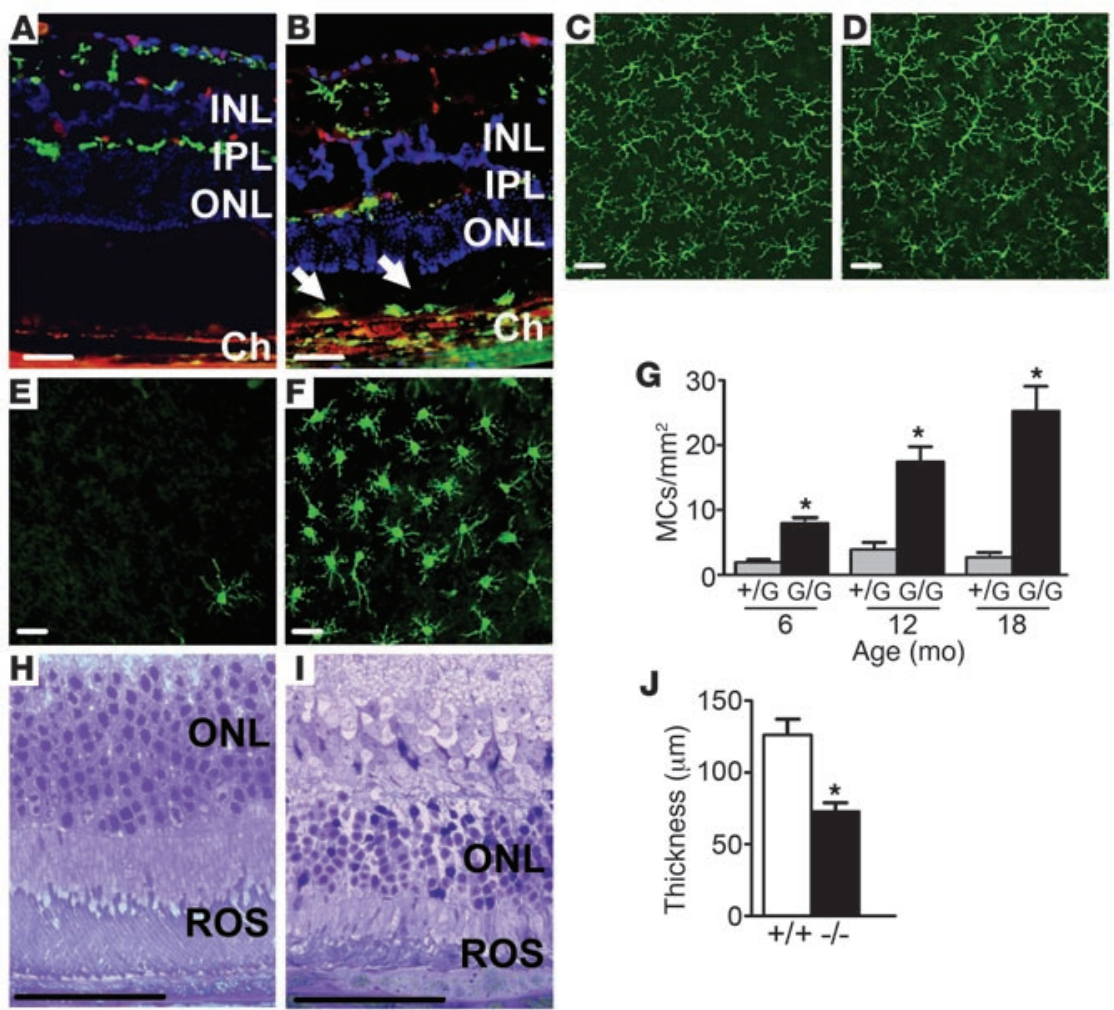

$\mathbf{J}$

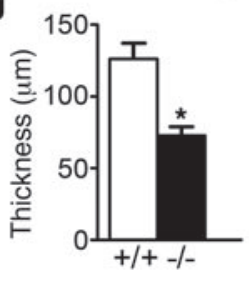

Figure 3

SrMCs accumulate in CX3CR1-1- C57BL/6 mice with age and lead to retinal degeneration. (A) Sections from an 18-month-old CX3CR1+/GFP mouse showed GFP-positive cells in close proximity to Griffonia simplicifolia-positive (red) retinal endothelial cells. (B) Sections from an 18-month-old CX3CR1 GFP/GFP mouse revealed the same distribution of GFP-positive cells in the inner retina but showed additional subretinal GFP-positive cells juxtaposed to the RPE cell layer (arrows). (C and D) Confocal images of the outer plexiform layer revealed that the entire network of ramified MCs was GFP positive and similarly dense in CX3CR $1+/$ GFP $(\mathbf{C})$ and CX3CR1 GFP/GFP mice (D). (E and F) RPE flatmounts of aged mice showed a strong accumulation of SrMCs in CX3CR1+/GFP (E) and CX3CR1 GFP/GFP mice (F). (G) Quantification of subretinal GFP-positive cells on RPE flatmounts revealed that SrMCs accumulated progressively in CX3CR1GFP/GFP mice and were significantly more numerous than in CX3CR1+/GFP mice at all time points. ( $H$ and I) Toluidine blue-stained epoxy retinal semithin sections showed degeneration of photoreceptors in 18-month-old CX3CR1 $1^{-/}$(I) mice compared with CX3CR1 $1^{+/+}(\mathbf{H})$ mice. (J) Measurements of photoreceptor cell layer thickness showed significant thinning of the photoreceptor cell layer in CX3CR1 $1^{-/}$mice compared with $\mathrm{CX} 3 \mathrm{CR} 1^{+/+}$mice. Experiments were performed on $4-8$ eyes from different mice per group. ${ }^{\star} P<0.05$. Ch, choroid. ROS, rod OS. Scale bars: $50 \mu \mathrm{m}$.

Drusen observed in CX3CR1 knockout mice are lipid-bloated SrMCs. Subretinal deposits are characteristic in AMD. Funduscopy of senescent $\mathrm{CX}_{3} \mathrm{CR}^{+/+}$(Figure 5A) and CX3CR1-/- (Figure 5B) mice showed that all the CX3CR1/-- mice had numerous drusen-like yellow nodular deposits in the deep layer of the retina that were not present in age-matched CX3CR $1^{+/+}$or 2 -month-old CX3CR1-/ mice (data not shown). Similar features were observed in a second strain of CX3CR1 knockout mouse, CX3CR1 GFP/GFP (23). To characterize in more detail the anatomical structure that produced this drusen-like appearance under funduscopy in CX3CR $1^{-/-}$mice, we flatmounted the eyes after removal of the neuroretina, thus exposing the RPE monolayer. The RPE monolayer of CX3CR $1^{+/+}$ animals, observed in tangential white light, appeared regular and smooth (Figure 5C). In contrast, in knockout mice, examination of the monolayer showed it was abutted by multiple elevated white spots (Figure 5D). Higher magnification revealed the intracellular location of this white material: bloated cells adjacent to the RPE cell monolayer. These intracellular yellowish-white deposits were present only in GFP-positive cells (Figure 5, E-G). Further confocal microscopy of these GFP-positive ramified cells (Figure $5 \mathrm{H}$ ) used the specific MC marker 5D4 (22) (Figure 5I) and CD11b or F4/80 (data not shown) to identify these cells as activated SrMCs. Not all SrMCs were bloated, but only the bloated cells appeared drusen-like on RPE flatmounts.

The presence of yellowish-white deposits in 5D4-, CD11b- and F4/80-positive SrMCs was corroborated in CX3CR1 ${ }^{-/}$eyes (data not shown). Histological sections of 12-month-old $\mathrm{CX} \mathrm{CR}^{+/+}$(Figure 3J) and CX3CR1 ${ }^{-/}$(Figure $5 \mathrm{~K})$ mice showed subretinal cells adhering to the pigmented monolayer of the RPE in CX3CR $1^{-/-}$but not CX3CR $1^{+/+}$mice. Ultrastructural analysis of senescent CX3CR1-/eyes by transmission electron microscopy confirmed the presence of subretinal cells between the outer segment (OS) and the RPE cells (Figure $5 \mathrm{~L}$ ), which contained intracellular lipid deposits and remnants of OS (Figure 5M), as typically observed in RPE cells (24). Rhodopsin immunoreactivity in CD11b-positive SrMCs of CX3CR1 $1^{-/}$RPE flatmounts (Figure $5 \mathrm{~N}$ ) was consistent with ingestion of OS by SrMCs.

SrMC accumulation is associated with CNV and retinal degeneration in CX3CR1 knockout mice. Laser coagulation of the mouse choroid induces a local inflammatory reaction composed of blood-derived macrophages and activated MCs (25). After detecting SrMC accumulation in senescent and ambient light-exposed CX3CR1-deficient mice, we studied the influence of CX3CR1 deficiency in laser injury. Fourteen days after a laser injury, CX3CR1 ${ }^{+/ G F P}$ mice showed some GFPpositive SrMCs adjacent to the choroid scar (Figure 6A), but many more such cells had accumulated in the CX3CR1 ${ }^{\mathrm{GFP} / \mathrm{GFP}}$ mice (Figure 6B) at this stage. The cell counts after laser impact (Figure 6C) showed a transient increase in the CX3CR $1^{+/ G F P}$ mice, peaking at day 7; again, the increase in CX3CR1 1 GFP/GFP mice was significantly higher and continued to rise. Immunohistochemistry at day 7 in CX3CR1 $1^{+/+}$mice (Figure 6, D-F) revealed that the proangiogenic factor VEGF (Figure 6D) was produced by activated SrMCs that expressed F4/80 (Figure 6, E and F).

At 14 days after laser injury, there was significantly less CNV in CX3CR1 $1^{+/+}$mice (Figure $6 \mathrm{G}$ ) than in their CX3CR1 $1^{-/-}$counterparts (Figure $6 \mathrm{H}$ ). Quantification of the surface covered by the endothelial cells showed twice as much neovascularization in the CX3CR1/- and CX3CR1 GFP/GFP knockout strains (Figure 6I) than their controls.

Finally, because retinal degeneration worsens secondary to subretinal neovascularization in $\mathrm{AMD}$, we tested whether the increased $\mathrm{MC}$ recruitment in the vicinity of the subretinal neovascularization 

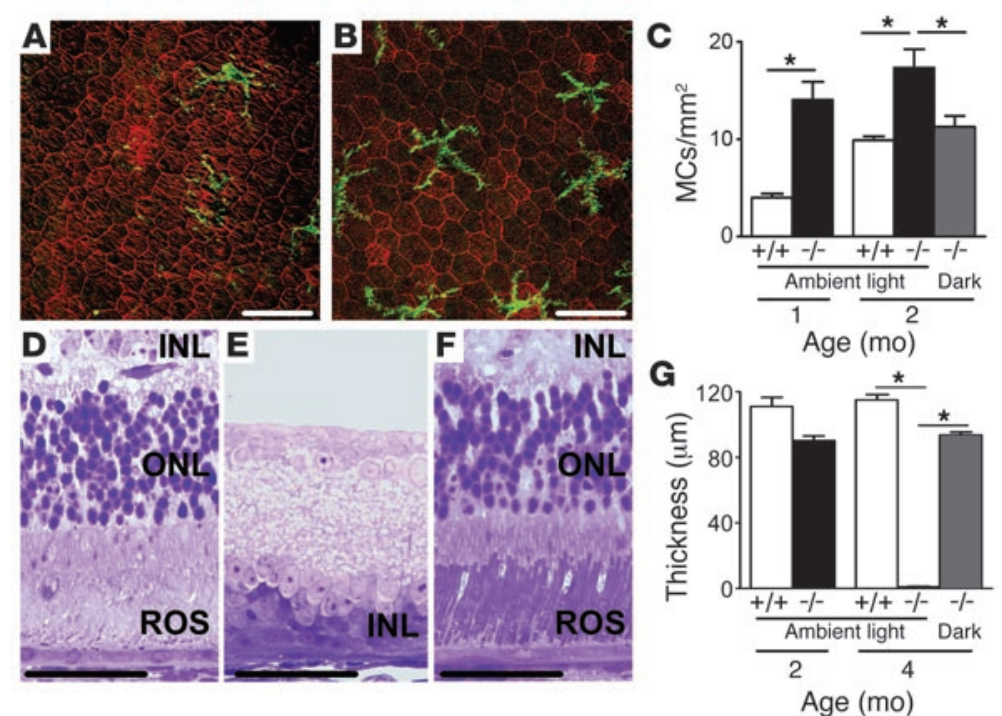

\section{Figure 4}

SrMC accumulation induces retinal degeneration in albino $\mathrm{CX} 3 \mathrm{CR} 1^{-1-}$ mice. (A and B) RPE flatmounts of albino CX3CR $1^{+/+}$BALB/C (A) and CX3CR1-/- BALB/C mice (B) showed more numerous CD11b-positive (green) SrMC abutting the RPE (Phalloidin, red) in CX3CR1-deficient animals. (C) Quantification of subretinal CD11b-positive cells on RPE flatmounts revealed a significantly higher density of MCs in CX3CR1 $1^{-1-}$ mice at 1 and 2 months of age raised in ambient light conditions. $\mathrm{CX} 3 \mathrm{CR} 1^{-1-} \mathrm{BALB} / \mathrm{c}$ mice raised in complete darkness showed significantly fewer SrMC than ambient light-raised CX3CR1 $1^{-/-}$BALB/c mice. (D-F) Toluidine blue-stained epoxy retinal semithin sections showed complete degeneration of all photoreceptors in albino $\mathrm{CX} 3 \mathrm{CR} 1^{-/-} \mathrm{BALB} / \mathrm{c}$ mice $(\mathrm{E})$ at 4 months of age compared with $\mathrm{CX} 3 \mathrm{CR} 1^{+/+} \mathrm{BALB} / \mathrm{c}$ mice (D). This degeneration was prevented in $\mathrm{CX} 3 \mathrm{CR} 1^{-/-}$ BALB/c mice raised in darkness $(\mathbf{F})$. (G) Measurements of photoreceptor cell layer thickness showed significant and progressive degeneration in albino CX3CR $1^{-/-}$ $\mathrm{BALB} / \mathrm{c}$ mice, which was completely reversed by raising CX3CR $1^{-/-} \mathrm{BALB} / \mathrm{c}$ mice in darkness. Experiments were performed on 8-10 eyes from different mice per group. ${ }^{\star} P<0.05$. Scale bars: $50 \mu \mathrm{m}$.

was associated with an aggravation of the retinal degeneration. In 12 -month-old animals, in which a $30 \%$ reduction in outer nuclear layer thickness was observed in CX3CR $1^{-/}$compared with $\mathrm{CX} 3 \mathrm{CR} 1^{+/+}$mice, we analyzed the photoreceptor cell layer at 21 days after the laser treatment. In $\mathrm{CX} 3 \mathrm{CR} 1^{+/+}$mice, photoreceptor cell layer thickness adjacent $(200 \mu \mathrm{m})$ to the laser-induced retinal destruction did not differ significantly from that in the nonimpacted retina (Figure $6 \mathrm{~J})$. In contrast, under these conditions, the photoreceptor layer had thinned substantially in CX3CR1/- mice (Figure 6K). This difference may result from the combination of age- and laser-induced degeneration. To evaluate the laser-induced alteration, the relative retinal thicknesses of injured and uninjured retina were compared at different distances from the laser impact (ranging 100-1,000 $\mu \mathrm{m}$ ). Laser-dependent retinal degeneration was significantly more pronounced in $\mathrm{CX} 3 \mathrm{CR} 1^{-/-}$mice than in $\mathrm{CX} 3 \mathrm{CR} 1^{+/+}$mice at distances of 100 and $200 \mu \mathrm{m}$ but not at 300 and $400 \mu \mathrm{m}$ (Figure 6L).

\section{Discussion}

Here we show that retinal MCs all and invariably expressed CX3CR1, that these cells accumulated subretinally in AMD, and that the T280M polymorphism of CX3CR1 was associated with both impaired cell migration and AMD. MCs accumulated under the retinas of CX3CR1-deficient mice with age, after laser injury, or with albino background. This accumulation elicited photore- ceptor degeneration and exacerbated neovascularization. Moreover, the resulting prolonged contact of MC with lipid-rich OS was associated with intracellular lipid accumulation in the MCs. Surprisingly, these subretinal microglial foam cells turned out to be the origin of drusen-like deposits in mice. Our results show that accumulation of MCs in the subretinal space may be a driving force in the pathogenesis of AMD, rather than a secondary occurrence.

Our study of patients with AMD showed that homozygosity for the M280 allele was consistently more frequent in groups with AMD than in studies of other populations and confirmed a previous report (10) of a dominant association between the CX3CR1 M280 allele and AMD risk. Subjects in the control studies were not checked for AMD, and some certainly had or will develop the disease. The effect of this bias, however, should be an underestimation of the impact of CX3CR1 polymorphisms on AMD risk. These data indicate that the chemokine receptor CX3CR1 may play an important role in AMD pathogenesis.

To understand how CX3CR1 polymorphisms contribute to AMD pathogenesis, it is essential to identify the types of cells expressing CX3CR 1 in the retina. Our immunohistochemical analysis in humans showed that CX3CR1 was expressed on all retinal MCs, which were identified by their morphology and CD18 expression. In addition, experiments using CX3CR1 $1^{+/ G F P}$ mice and the murine MC markers CD11b, F4/80, and 5D4 confirmed that only MCs express CX3CR1. Using both techniques in 2 different species, we found no CX3CR1 expression in neurons, astrocytes or glial cells: as a previous report showed for the CNS (14), MCs are the only cells in the retina that express CX3CR1. Contrary to a previous report (10), when we used the CX3CR1-specific antibody revealed by the fast red system to circumvent autofluorescence interference and conducted experiments with a reporter gene, we failed to find significant CX3CR1 expression in RPE cells. In AMD, CX3CR1-positive MCs accumulated subretinally in affected areas of the macula. Our data are consistent with the infiltration of the subretinal space by activated MCs previously described in AMD (15) and indicate that a specific inflammatory environment characterized by production of specific cytokines and chemokines may determine particular pathological conditions.

Cells of the CNS and of the retina express CX3CL1 abundantly $(26,27)$. On the other hand, RPE cells under oxidative stress express other monocyte chemoattractant chemokines such as CCL2 (28), which may be responsible for the recruitment of MCs in the subretinal space. To evaluate the effect of the M280 polymorphism on cell migration in a CX3CL1-rich environment, we tested M280 CX3CR1 monocyte migration through a CX3CL1-coated porous membrane in response to CCL2. Previous studies show that the M280 polymorphism provokes loss of chemotaxis (17) or increases adherence to its ligand (19). Here, the presence of bound CX3CL1 significantly inhibited the CCL2-dependent chemotaxis of monocytes expressing the CX3CR1 M280 variant (Figure 2). If similar alterations occur in vivo to retinal MCs, the M280 polymorphism may cause excessive MC adherence to membrane-anchored CX3CL1 and reduce migration in response to other inflammatory chemoattractants. Over the years, in subjects with the M280 polymorphism, clearance of MCs from the subretinal space (in response 

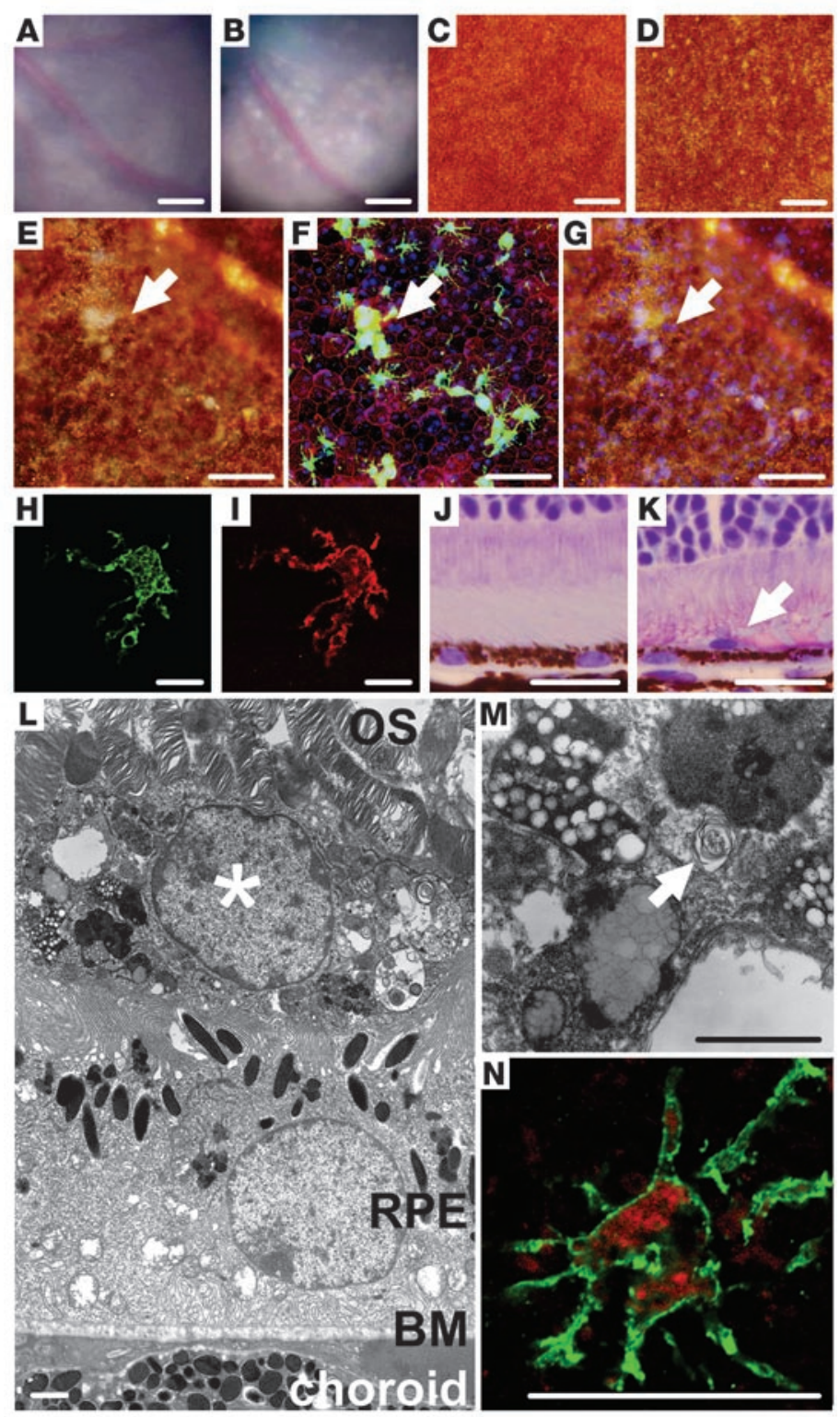

to soluble CX3CL1 or other chemoattractants) would thereby be inhibited, and SrMC accumulation might occur.

To simulate CX3CR1 dysfunction in an animal model, we used 2 independently generated CX3CR1 knockout mouse strains. Our data demonstrated strong and persistent SrMC accumulation in CX3CR1-deficient mice in 3 experimental conditions: laser injury, aging, and albino animals. In contrast, control mice with laser injury only transiently amassed ramified F4/80- and GFP-positive cells, and aging and albino control animals slowly or steadily accumulated SrMCs. We and others have previously demonstrated that CX3CR1 deficiency reduces macrophage aggregation in atherosclerotic lesions $(20,29)$, probably by limiting monocyte recruitment from the blood. In view of the role of CX3CR1 in monocyte recruitment, the excessive number of MCs observed in CX3CR1-deficient mice is unlikely to be the result of an excessive influx of monocytes.

Unlike the monocytes in atherosclerotic lesions, MCs can proliferate in situ (30). Their accumulation may be the result of exces-

\section{Figure 5}

Drusen observed in CX3CR1 knockout animals are bloated SrMCs. (A-D) Comparison of fundus photos and micrographs of a RPE flatmount in tangential light of 1 -year-old CX3CR $1^{+/+}(\mathbf{A}$ and $\mathbf{C})$ and CX3CR $1^{-1-}$ mice (B and $\mathbf{D}$ ) revealed multiple drusen in CX3CR1 $1^{-1-}$ mice. (E-G) Higher-magnification image of a drusen (arrow) in a CX3CR1GFP/GFP mouse in tangential light (E) superimposed with GFP-positive (green; red, Phalloidin; blue, DAPI) subretinal ramified cells (F) on RPE flatmounts. Merge of tangential light and DAPI is shown in $\mathbf{G}$. (H and I) GFP-positive subretinal ramified cells $(\mathbf{H})$ on CX3CR1GFP/GFP RPE flatmounts were positive for the MC marker 5D4 (red, I). ( $\mathbf{J}$ and $\mathbf{K}$ ) Histological sections of historesin-embedded 12-month-old $\mathrm{CX} 3 \mathrm{CR} 1^{+/+}(\mathbf{J})$ and $\mathrm{CX} 3 \mathrm{CR} 1^{-/-}(\mathbf{K})$ eyes showed subretinal cells abutting the RPE in $\mathrm{CX}_{3} \mathrm{CR} 1^{-1-}(\mathbf{K}$, arrow) but not in CX3CR $1^{+++}$mice. (L) Electron microscopy of the RPE/OS interface in $\mathrm{CX} 3 \mathrm{CR} 1^{-/}$mice revealed subretinal cells (asterisk) with multiple phagosomes juxtaposed to the RPE cell layer. (M) Higher magnification of these subretinal cells revealed multiple lipid deposits and typical remnants of rod OSs in phagosomes (arrow). (N) Subretinal CD11b-positive (green) dendritic cells contained rhodopsin-positive inclusions (red) in confocal micrographs of RPE flatmounts from $\mathrm{CX} 3 \mathrm{CR} 1^{-1-}$ mice. Results are representative of at least 3 independent experiments. Scale bars: $500 \mu \mathrm{m}$ (A-D), $50 \mu \mathrm{m}$ (E-G), $20 \mu \mathrm{m}$ $(\mathbf{H}-\mathbf{K}$ and $\mathbf{N}), 1 \mu \mathrm{m}$ ( $\mathbf{L}$ and $\mathbf{M})$.

sive proliferation or inhibition of their clearance from the subretinal space. CX3CL1, however, has been shown to facilitate microglia proliferation (31), and excessive proliferation is unlikely to occur in CX3CR1 deficiency. On the other hand, SrMC clearance by apoptosis or migration (egression) may be affected by abrogation of CX3CR1. This question is currently under investigation in our laboratory.

Interestingly, CCL2 and CCR2 signaling did not appear to be involved in retinal MC distribution. Although CCL2 ${ }^{--}$(and CCR2-/-) mice have previously been shown to develop signs of AMD secondary to a macrophage recruitment deficit (11), they did not develop significant SrMC accumulation $(1.7 \pm 0.2$ SrMCs $/ \mathrm{mm}^{2}$ ) at 12 months of age in the present study. The macrophage-dependent debris clearance (11) may play a role only at a more advanced stage of the disease.

In CX3CR1-deficient mice, the resulting prolonged presence of MCs in the subretinal space was associated with excessive OS phagocytosis by SrMCs, which subsequently accumulated intracellular lipids. These subretinal microglial foam cells were the origin of the drusen-like deposits in mice. Similarly, in humans, we found CX3CR1-positive bloated SrMCs in the eyes of individuals with AMD (Figure 1B), and others have previously shown that clearance of photoreceptor debris by activated MCs causes bloated SrMCs (15). It is tempting to speculate that SrMCs contribute to some extent to the drusen formation in AMD and that the rounded contours and steeply sloping sides characteristic of drusen (5) may derive in part from degenerating bloated MCs that are subsequently covered by RPE cells. Supporting this theory is the fact that drusen contain numerous degenerating organelles, the origin of which may be retinal MCs (32). Moreover, CX3CR1 deposits were found in drusen (Figure 1F). Similar drusen deposits have previously been reported to contain apolipoprotein E, complement factors, MHC, and amyloid oligomers, among others $(5,33-36)$. When activated, MCs can express ApoE (37), complement factors (38), MHC (39), and the $\beta$-amyloid precursor protein (40) as well as CX3CR1. MCs can be a major source of oxidative stress through respiratory bursts 

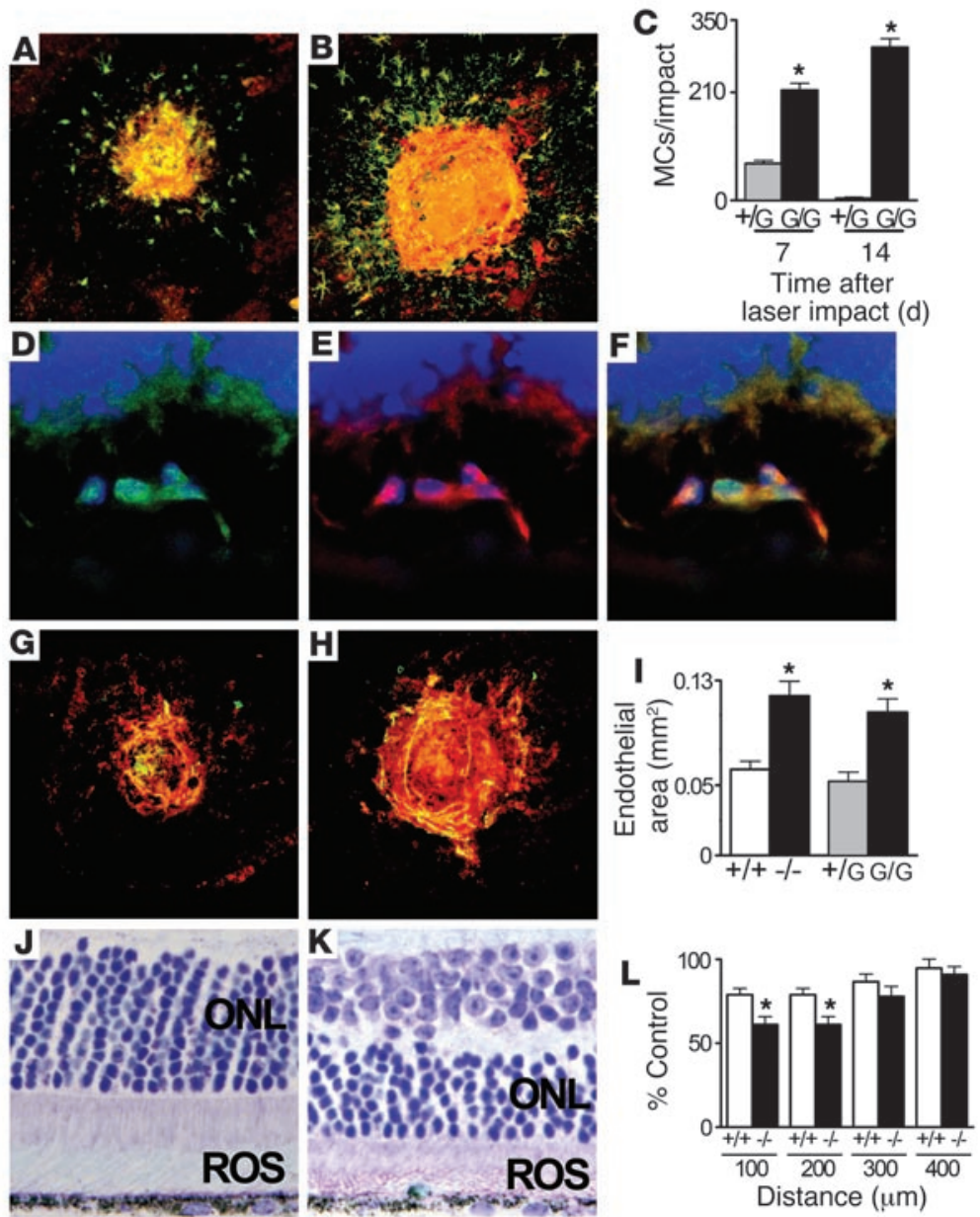

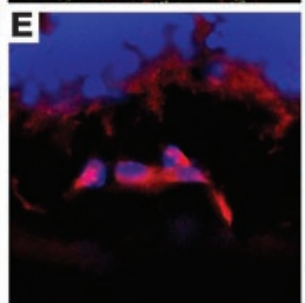

\section{Figure 6}

SrMCs accumulate after laser injury, exacerbate neovascularization, and induce adjacent retinal degeneration. (A and B) At 3 months of age, CX3CR1+/GFP mice displayed some GFP-positive (green) SrMCs adjacent to the CNV (red, Griffonia simplicifolia) 14 days after laser injury (A), and CX3CR1GFP/GFP mice showed strong SrMC accumulation (B). (C) CX3CR1+/GFP mice showed a transient increase of SrMCs after laser impact, peaking at day 7; however, SrMCs in CX3CR1GFP/GFP mice were significantly more numerous at that time and continued to accumulate. (D-F) Immunohistochemistry at day 7 revealed VEGF expression (green) in F4/80-positive activated CX3CR $1^{+/+}$ MCs (red) in the subretinal space. Nuclei were stained with DAPI (blue). ( $\mathbf{G}$ and $\mathbf{H}$ ) Micrographs of fluorescein dextran (green) perfused RPE/choroidal flatmounts double labeled with endothelial cell marker Griffonia simplicifolia (red) 14 days after laser impact in $\mathrm{CX} 3 \mathrm{CR} 1^{+/+}(\mathbf{G})$ and $\mathrm{CX} 3 \mathrm{CR} 1^{-/-}$ (H) mice showed representative CNV. (I) Quantification showed exacerbated neovascularization in the knockout strains. (J) Eyes of CX3CR1 ${ }^{+/+}$mice (12 months old) showed a normal photoreceptor cell layer $200 \mu \mathrm{m}$ adjacent to the impact 21 days after laser treatment. (K) At this stage CX3CR1-/- mice displayed SrMCs and a thinned and irregular photoreceptor cell layer. (L) Laser-dependent retinal degeneration (calculated as described in Methods) was significantly more pronounced in $\mathrm{CX} 3 \mathrm{CR} 1^{-1-}$ mice only at 100 and $200 \mu \mathrm{m}$. Experiments were performed on 8-10 eyes from different mice per group. ${ }^{\star} P<0.05$. Scale bars: $50 \mu \mathrm{m}(\mathbf{A}, \mathbf{B}, \mathbf{G}, \mathbf{H}, \mathbf{J}$, and $\mathbf{K}), 10 \mu \mathrm{m}(\mathbf{D}-\mathbf{F})$.

neovascularization after laser injury. To recapitulate, SrMC accumulation in CX3CR1-deficient mice is associated with photoreceptor degeneration in the absence of any primary photoreceptor or RPE disease. To our knowledge, no such mechanism of retinal degenera-

(41) that can cause the oxidative protein and lipid modifications associated with drusen. The inflammatory proteins found in drusen may therefore be of retinal MC origin, at least in part.

Another consequence of the prolonged presence of MCs in the subretinal space may be photoreceptor cell death. In CX3CR1-1mice, retinal degeneration was observed in senescent mice, in albino mice, and in the vicinity of subretinal neovascularization. The retinal degeneration in $\mathrm{CX} 3 \mathrm{CR} 1^{-/}$mice was always preceded by SrMC accumulation and could be averted by limiting MC accumulation in the subretinal space, as seen in CX3CR1-/- albino mice raised in the dark (Figure 4). SrMC accumulation was observed as early as 6 months of age in pigmented and 1 month in albino CX3CR1-deficient mice, before any significant retinal degeneration (data not shown). There are no naturally occurring mutations known to cause retinal degeneration in $\mathrm{BALB} / \mathrm{c}$ mice, and the time course of the degeneration, starting at 2 months, did not match any known retinal degeneration caused by photoreceptor or RPE gene defects. We further note that neither photoreceptors nor RPE cells contained express CX3CR1. Activated MCs participate in ganglion cell death in vivo (42) and can induce photoreceptor cell death in vitro (43). Neuronal cell toxicity caused by the prolonged presence of MCs (CX3CR1 1 GFP/GFP) in the brain has previously been described as a mechanism of neurodegenerative diseases (14), and similar mechanisms may cause the degeneration observed in the $\mathrm{CX} 3 \mathrm{CR} 1^{-/-} \mathrm{BALB} / \mathrm{c}$ mice, in the senescent $\mathrm{CX} 3 \mathrm{CR} 1^{-/-} \mathrm{C} 57 \mathrm{BL} / 6$ mice, and in the vicinity of subretinal tion has previously been described.

Furthermore, we showed that activated SrMCs adjacent to the laser injury site express VEGF. In CX3CR1-deficient mice, the increased accumulation of SrMCs and subsequent secretion of VEGF may be responsible for the development of the exaggerated neovascularization. This is consistent with our previous work, which showed that MCs can develop proangiogenic properties in the retina (44). Moreover, CX3CR1-positive MCs were found in human CNV, which indicates that SrMCs are a possible cause or aggravating factor for CNV in AMD.

In summary, our results suggest that $\mathrm{MC}$ accumulation in the subretinal space may be a driving force in the pathogenesis of AMD and not a mere consequence of primary RPE or photoreceptor disease. Our study challenges the long-standing paradigm of drusen genesis as the gradual accumulation of extracellular debris from the RPE and choroid. We propose that SrMC accumulation, resulting from a migratory defect associated with CX3CR1, plays a key role in drusen formation, $\mathrm{CNV}$, and retinal degeneration, the main features of AMD.

\section{Methods}

Study participants and AMD assessment. The GAMD study included 285 participants (29\% men) recruited in the "Centre d'angiographie et de laser" during the second trimester of 2006. AMD was diagnosed in all participants by 2 independent ophthalmologists. The DETER study included 520 blood donors (56\% men) recruited during 2003-2005 at the blood bank of the 
Hospitalier Pitié-Salpétrière (P. Deterre, unpublished observations). In accordance with the Declaration of Helsinki, patients and volunteers provided written and informed consent for the GAMD and DETER studies, which were approved by the Pitié-Salpétrière and Hotel-Dieu (Paris, France) ethics committees (no. 0611303). Genomic DNA was extracted from EDTA anticoagulated venous blood with the DNeasy kit (Qiagen), following the manufacturer's standard protocols. Previously published studies were used as references: the ECTIM study (16), the Offspring Cohort of the FHS (17), and the GENIC study (18). The ECTIM study is a case-control study specially designed to identify genetic variants associated with myocardial infarction. The study targeted 4 populations covered by CHD registries (WHO/ MONICA): 3 in France, in the regions of Lille, Strasbourg, and Toulouse, and 1 in Northern Ireland, in the region of Belfast. GENIC is a case-control study of cerebrovascular stroke, conducted in collaboration with a French network of specialized clinical departments. The Offspring Cohort of the FHS began in 1971, enrolling 5,124 children and children-in-law of the original participants. They undergo a comprehensive medical exam every 4 years (defined as a cycle). The FHS Offspring Cohort contained 1,888 unrelated participants for whom DNA was available. Of these, 1,861 had given informed consent for genotyping and were entered into the present study.

Screening for polymorphisms. CX3CR1 polymorphisms were identified with Minor Groove Binder PCR-based amplification technology from PerkinElmer (Applied Biosystems), as described elsewhere (45).

Human eye sections. Eyes from 4 patients with AMD ( 3 dry and 1 wet form) and 3 age-matched control donors were obtained from the eye bank of Quebec. The maculae were dissected, fixed in PBS with 4\% paraformaldehyde (PFA), frozen, and sectioned $(10 \mu \mathrm{m})$. Patients and volunteers gave informed consent in accordance with Quebec's ethics regulations.

Chemotactic migration assay. PBMCs were isolated from heparinized venous blood from healthy volunteer individuals by 1 -step centrifugation on a Ficoll separating solution (Eurobio), washed twice with PBS, and resuspended at $5 \times 10^{6}$ cells $/ \mathrm{ml}$ in serum-free DMEM (Invitrogen) containing $5 \mathrm{mg} / \mathrm{ml}$ of bovine serum albumin. Chemotaxis was assayed in a 24 -well chemotaxis chamber through transwell filters (Corning Incorporated). Filters (8- $\mu \mathrm{m}$ pore size) were treated with anti-histidine antibody $(5 \mu \mathrm{g} / \mathrm{ml})$ for 2 hours at room temperature, washed with PBS, and coated with CX3CL1-Histidine $(0.1 \mu \mathrm{g} /$ $\mathrm{ml}$ ) or PBS overnight at $4^{\circ} \mathrm{C}$. After the filters were rinsed with PBS, $100 \mu \mathrm{l}$ of cell suspension was loaded onto the filter. CCL2 (100 nM) or PBS was placed in the lower chamber. The plate was then incubated for $3 \mathrm{~h}$ at $37^{\circ} \mathrm{C}, 100 \%$ humidity, and $5 \% \mathrm{CO}_{2}$. Monocytes that had migrated through the bottom surface of the filters were then counted by flow cytometry (FACSCalibur; BD) based on CD14 expression (BD Biosciences). The mean number of monocytes in triplicate samples was determined, and a chemotactic index - or ratio of cells in the lower chamber with and without CCL2 - was calculated for monocytes migrating through filters with or without a CX3CL1 coating.

Animals. CX3CR1/- and CX3CR1 $1^{\mathrm{GFP} / \mathrm{GFP}}$ mouse strains on C57BL/6 background and their controls were generated as described previously (20, 23). $\mathrm{CX} 3 \mathrm{CR} 1^{-/-} \mathrm{C} 57 \mathrm{BL} / 6$ mice were backcrossed for 6 generations into the BALB/c background (Janvier) to obtain the CX3CR1 $1 /-\mathrm{BALB} / \mathrm{c}$ strain and kept in specific pathogen-free conditions with food and water available ad libitum and housed in a 12-h light/12-h dark (100-500 lux) cycle or in complete darkness (CX3CR1/- BALB/c). Animal experiments were approved by the local Institutional Animal Care and Use Committee: Centre d'Exploration Fonctionelle, Pitié-Salpétrière.

Fundus photography and laser-coagulation. Mice were anesthetized by intramuscular injection of ketamine $(50 \mathrm{mg} / \mathrm{kg})$ and xylazine $(10 \mathrm{mg} / \mathrm{kg})$. Pupils were fully dilated with $1 \%$ tropicamide or $1 \%$ atropin. Coverslips positioned on the mouse cornea were used as a contact glass. Fundus photographs were taken with a digital camera mounted on an ophthalmic operating microscope (Zeiss). Laser coagulations were performed 1-2 disc diameters away from the papillae with an Argon laser $(532 \mathrm{~nm})$ mounted on a slit lamp (400 mW, $50 \mathrm{~ms}$ and $50 \mu \mathrm{m})$.

Immunohistochemistry. Frozen sections were stained according to previously described standard immunohistochemical procedures (44). Primary antibodies used were rabbit polyclonal anti-human CX3CR1 (Chemicon), mouse anti-human CD18 (BD Biosciences - Pharmingen), rat monoclonal anti-CD11b (Serotec), rat anti-F4/80 (BD Biosciences - Pharmingen), mouse anti-rat keratan sulfate proteoglycan (5D4, Seikagaku), mouse anti-GFP (Torrey Pines Biolabs), VEGF (Chemicon), and goat anti-mouse CX3CL1 (R\&D). Lectins used were TRITC-conjugated Lectin Ulex Europaeus or Griffonia simplicifolia (Sigma-Aldrich) and Rhodamine Phalloidin (Invitrogen). The corresponding Alexa or alkaline phosphatase-conjugated secondary antibodies (Invitrogen) were used to reveal the primary antibodies, and sections were counterstained with DAPI or hemalun. Alkaline phosphatase was revealed with fast red (Sigma-Aldrich). Sections and flatmounts were viewed with a fluorescence microscope (BX51; Olympus) or a confocal microscope (LSM 510 laser scanning microscope; Zeiss). All immunostaining was repeated at least 3 times, and staining that omitted the primary antibody served as a negative control.

Choroidal flatmounts and SrMC/CNV quantifications. Eyes were enucleated, fixed in 4\% PFA for 15 minutes at room temperature, and sectioned at the limbus; the cornea and lens were discarded (for $\mathrm{CNV}$, mice were perfused with Fluorescein Dextran $10^{6}$ before enucleation). The retinas were carefully peeled from the RPE/choroid/sclera. Retinas and choroids were fixed for an additional 15 minutes in methanol at $-20^{\circ} \mathrm{C}$ and incubated with the indicated primary and secondary antibodies and lectins. The choroids and retinas were radially incised, flatmounted, and viewed with a fluorescence microscope (BX51; Olympus). For RPE flatmount photographs, tangential white light was applied by placing the light-conducting glass fibers of a KL2500LCD Schott lamp directly over the coverslip, next to the objective. SrMC were counted on GFP or F4/80 stained whole RPE/choroidal flatmounts up to the ciliary body and on the OS side of the retina. The surface of the RPE was measured and SrMC density calculated. CNV was measured on photographs with Image J analysis software. Retinal degeneration was assessed on historesin-embedded sections (see below) that crossed the center of the laser impact.

Histology and electron microscopy. For histology, eyes were fixed in $0.5 \%$ glutaraldehyde, 4\% PFA PBS for $2 \mathrm{~h}$, dehydrated, and mounted in historesin. Sections $(5 \mu \mathrm{m})$ were cut and stained with eosin. For electron microscopy and semithin sections, eyes were fixed in $2.5 \%$ glutaraldehyde of cacodylate buffer (0.1 M, pH 7.4). After 1 hour, eyeballs were dissected, fixed for another 3 hours, postfixed in $1 \%$ osmium tetroxide in cacodylate buffer, and dehydrated in graduated ethanol solution. The samples were included in epoxy resin and oriented. Semithin sections $(1 \mu \mathrm{m})$ were sliced with an ultramicrotome Reichert Ultracut E (Leica), stained by toluidine blue, and examined with a light microscope to measure the photoreceptor layer thickness. Multiple measurements of the photoreceptor cell layer of injured and uninjured retina were used to calculate the relative retinal thicknesses at sites $100-1,000 \mu \mathrm{m}$ from the site of laser impact. Ultrathin sections (80 $\mathrm{nm}$ ) were stained for contrast with uranyl acetate and lead citrate and were observed in a JEOL 100 CX II electron microscope (JEOL) with $80 \mathrm{kV}$. The absolute values of outer nuclear layer thickness of epoxy-embedded semithin and historesin-embedded sections were not comparable because of stronger shrinkage for the former.

Statistics. Allelic frequencies were calculated by gene counting. Hardy-Weinberg equilibrium was tested with the $\chi^{2}$ test with 1 degree of freedom. We used the $\chi^{2}$ test to compare genotype distributions and allele frequencies in participants. Statistical comparisons used unpaired 2-sample $t$ tests for means and Mann-Whitney U tests. All analyses and graphic representation were performed with Prism software (version 4.0c; GraphPad Software), and values are reported as mean \pm SEM. A $P$ value less than 0.05 was considered significant. 


\section{Acknowledgments}

The authors wish to thank Arnaud Cangialosi, Christophe Klein, and Stéphanie Girard for technical assistance and Myriam Gort (Centre d'Exploration Fonctionnelle animal facility, Pitié-Salpétrière). We thank D. Littman and S. Jung for sharing the CX3CR1Kin GFP mice and R.S. Molday for the anti-rhodospsin antibody. This work was supported by grants from INSERM, by ANR grant "Cardiovasculaire, obésité et diabète" (AO5088DS), by ANR grant "blanc" (AO5120DD), by European Grant "Innochem" 518167, by PNR vision (AO6004SP), and by the FONDATION NRJ-Institut de France. C. Combadière is the recipient of a contract "Interface" from Assistance Publiques-Hopitaux de Paris, and M. Rodéro is supported by a studentship from the Canceropole Île de France.
Received for publication February 2, 2007, and accepted in revised form June 26, 2007.

Address correspondence to: Florian Sennlaub, INSERM U872, Centre de recherche des Cordeliers, 15 rue de l'Ecole de Médecine, 75006 Paris, France. Phone: 33-1-40-46-78-63; Fax: 33-1-40-4678-65; E-mail: sennlaub@idf.inserm.fr. Or to: Christophe Combadière, INSERM U543, Faculté de Médecine Pitié-Salpétrière, 91 Boulevard de l'Hôpital, 75013 Paris, France. Phone: 33-1-40-77-9892; Fax: 33-1-40-77-97-34; E-mail: combad@ccr.jussieu.fr.

Christophe Combadière, Charles Feumi, and William Raoul contributed equally to this work.
1. Friedman, D.S., et al. 2004. Prevalence of age-related macular degeneration in the United States. Arch. Ophthalmol. 122:564-572.

2. Sarks, S.H. 1976. Ageing and degeneration in the macular region: a clinico-pathological study. $\mathrm{Br}$. J. Ophthalmol. 60:324-341.

3. Bird, A.C., et al. 1995. An international classification and grading system for age-related maculopathy and age-related macular degeneration. The International ARM Epidemiological Study Group. Surv. Ophthalmol. 39:367-374.

4. Farkas, T.G., Sylvester, V., Archer, D., and Altona, M. 1971. The histochemistry of drusen. Am.J. Ophthalmol. 71:1206-1215.

5. Hageman, G.S., et al. 2001. An integrated hypothesis that considers drusen as biomarkers of immunemediated processes at the RPE-Bruch's membrane interface in aging and age-related macular degeneration. Prog. Retin. Eye Res. 20:705-732.

6. Souied, E.H., et al. 1998. The epsilon4 allele of the apolipoprotein $\mathrm{E}$ gene as a potential protective factor for exudative age-related macular degeneration. Am. J. Ophthalmol. 125:353-359.

7. Kimura, K., Isashiki, Y., Sonoda, S., Kakiuchi-Matsumoto, T., and Ohba, N. 2000. Genetic association of manganese superoxide dismutase with exudative age-related macular degeneration. Am. J. Ophthalmol. 130:769-773.

8. Haines, J.L., et al. 2005. Complement factor $\mathrm{H}$ variant increases the risk of age-related macular degeneration. Science. 308:419-421.

9. Edwards, A.O., et al. 2005. Complement factor $\mathrm{H}$ polymorphism and age-related macular degeneration. Science. 308:421-424.

10. Tuo, J., et al. 2004. The involvement of sequence variation and expression of CX3CR1 in the pathogenesis of age-related macular degeneration. FASEB J. 18:1297-1299.

11. Ambati, J., et al. 2003. An animal model of agerelated macular degeneration in senescent Ccl-2- or Ccr-2-deficient mice. Nat. Med. 9:1390-1397.

12. Tsutsumi, C., et al. 2003. The critical role of ocular-infiltrating macrophages in the development of choroidal neovascularization. J. Leukoc. Biol. 74:25-32.

13. Geissmann, F., Jung, S., and Littman, D.R. 2003. Blood monocytes consist of two principal subsets with distinct migratory properties. Immunity. 19:71-82.

14. Cardona, A.E., et al. 2006. Control of microglial neurotoxicity by the fractalkine receptor. Nat. Newrosci. 9:917-924.

15. Gupta, N., Brown, K.E., and Milam, A.H. 2003. Activated microglia in human retinitis pigmentosa, late-onset retinal degeneration, and age-related macular degeneration. Exp. Eye Res. 76:463-471.

16. Georges, J.L., et al. 2001. Interleukin- 6 gene polymorphisms and susceptibility to myocardial infarction: the ECTIM study. Etude Cas Temoin de l'Infarctus du Myocarde. J. Mol. Med. 79:300-305.
17. Kannel, W.B., Feinleib, M., McNamara, P.M., Garrison, R.J., and Castelli, W.P. 1979. An investigation of coronary heart disease in families: the Framingham offspring study. Am. J. Epidemiol. 110:291-290.

18. Touboul, P.-J., et al. 2000. Common carotid artery intima-media thickness and brain infarction: the Etude du Profil Génétique de l'Infarctus Cérébral (GENIC) case-control study. Circulation. 102:313-318.

19. Daoudi, M., et al. 2004. Enhanced adhesive capacities of the naturally occurring Ile249-Met280 variant of the chemokine receptor CX3CR1. J. Biol. Chem. 279:19649-19657.

20. Combadiere, C., et al. 2003. Decreased atherosclerotic lesion formation in CX3CR1/apolipoprotein E double knockout mice. Circulation. 107:1009-1016.

21. Ng, T.F., and Streilein, J.W. 2001. Light-induced migration of retinal microglia into the subretinal space. Invest. Ophthalmol. Vis. Sci. 42:3301-3310.

22. Margrain, T.H., Boulton, M., Marshall, J., and Sliney, D.H. 2004. Do blue light filters confer protection against age-related macular degeneration? Prog. Retin. Eye Res. 23:523-531.

23. Jung, S., et al. 2000. Analysis of fractalkine receptor CX(3)CR1 function by targeted deletion and green fluorescent protein reporter gene insertion. $\mathrm{Mol}$. Cell. Biol. 20:4106-4114.

24. Tamai, M., and O'Brien, P.J. 1979. Retinal dystrophy in the RCS rat: in vivo and in vitro studies of phagocytic action of the pigment epithelium on the shed rod outer segments. Exp. Eye Res. 28:399-411.

25. Caicedo, A., Espinosa-Heidmann, D.G., Pina, Y., Hernandez, E.P., and Cousins, S.W. 2005. Bloodderived macrophages infiltrate the retina and activate Muller glial cells under experimental choroidal neovascularization. Exp. Eye Res. 81:38-47.

26. Nishiyori, A., et al. 1998. Localization of fractalkine and CX3CR1 mRNAs in rat brain: does fractalkine play a role in signaling from neuron to microglia? FEBS Lett. 429:167-172.

27. Silverman, M.D., et al. 2003. Constitutive and inflammatory mediator-regulated fractalkine expression in human ocular tissues and cultured cells. Invest. Ophthalmol. Vis. Sci. 44:1608-1615.

28. Higgins, G.T., Wang, J.H., Dockery, P., Cleary, P.E., and Redmond, H.P. 2003. Induction of angiogenic cytokine expression in cultured RPE by ingestion of oxidized photoreceptor outer segments. Invest. Ophthalmol. Vis. Sci. 44:1775-1782.

29. Lesnik, P., Haskell, C.A., and Charo, I.F. 2003. Decreased atherosclerosis in CX3CR $1^{-/-}$mice reveals a role for fractalkine in atherogenesis. J. Clin. Invest. 111:333-340. doi:10.1172/JCI200315555.

30. Zeiss, C.J., and Johnson, E.A. 2004. Proliferation of microglia, but not photoreceptors, in the outer nuclear layer of the rd-1 mouse. Invest. Ophthalmol. Vis. Sci. 45:971-976.

31. Hatori, K., Nagai, A., Heisel, R., Ryu, J.K., and Kim, S.U. 2002. Fractalkine and fractalkine receptors in human neurons and glial cells. J. Neurosci. Res. 69:418-426.

32. Farkas, T.G., Sylvester, V., and Archer, D. 1971.
The ultrastructure of drusen. Am. J. Ophthalmol. 71:1196-1205.

33. van der Schaft, T.L., Mooy, C.M., de Bruijn, W.C., and de Jong, P.T. 1993. Early stages of age-related macular degeneration: an immunofluorescence and electron microscopy study. Br. J. Ophthalmol. 77:657-661.

34. Mullins, R.F., Russell, S.R., Anderson, D.H., and Hageman, G.S. 2000. Drusen associated with aging and age-related macular degeneration contain proteins common to extracellular deposits associated with atherosclerosis, elastosis, amyloidosis, and dense deposit disease. FASEB J. 14:835-846.

35. Luibl, V., et al. 2006. Drusen deposits associated with aging and age-related macular degeneration contain nonfibrillar amyloid oligomers. J. Clin. Invest. 116:378-385. doi:10.1172/JCI25843.

36. Johnson, L.V., et al. 2002. The Alzheimer's A betapeptide is deposited at sites of complement activation in pathologic deposits associated with aging and age-related macular degeneration. Proc. Natl. Acad. Sci. U. S. A. 99:11830-11835.

37. Xu, Q., et al. 2006. Profile and regulation of apolipoprotein E (ApoE) expression in the CNS in mice with targeting of green fluorescent protein gene to the ApoE locus. J. Neurosci. 26:4985-4994.

38. Bellander, B.M., Bendel, O., Von Euler, G., Ohlsson, M., and Svensson, M. 2004. Activation of microglial cells and complement following traumatic injury in rat entorhinal-hippocampal slice cultures. J. Neurotrauma. 21:605-615.

39. Matsubara, T., Pararajasegaram, G., Wu, G.S., and Rao, N.A. 1999. Retinal microglia differentially express phenotypic markers of antigen-presenting cells in vitro. Invest. Ophthalmol. Vis. Sci. 40:3186-3193.

40. Haass, C., Hung, A.Y., and Selkoe, D.J. 1991. Processing of beta-amyloid precursor protein in microglia and astrocytes favors an internal localization over constitutive secretion. J. Neurosci. 11:3783-3793.

41. Klegeris, A., and McGeer, P.L. 1994. Rat brain microglia and peritoneal macrophages show similar responses to respiratory burst stimulants. J. Neuroimmunol. 53:83-90.

42. Thanos, S., Mey, J., and Wild, M. 1993. Treatment of the adult retina with microglia-suppressing factors retards axotomy-induced neuronal degradation and enhances axonal regeneration in vivo and in vitro. J. Neurosci. 13:455-466.

43. Roque, R.S., Rosales, A.A., Jingjing, L., Agarwal, N., and Al-Ubaidi, M.R. 1999. Retina-derived microglial cells induce photoreceptor cell death in vitro. Brain Res. 836:110-119.

44. Checchin, D., Sennlaub, F., Levavasseur, E., Leduc, M., and Chemtob, S. 2006. Potential role of microglia in retinal blood vessel formation. Invest. Ophthalmol. Vis. Sci. 47:3595-3602.

45. Lavergne, E., et al. 2004. Intratumoral CC chemokine ligand 5 overexpression delays tumor growth and increases tumor cell infiltration. J. Immunol. 173:3755-3762. 